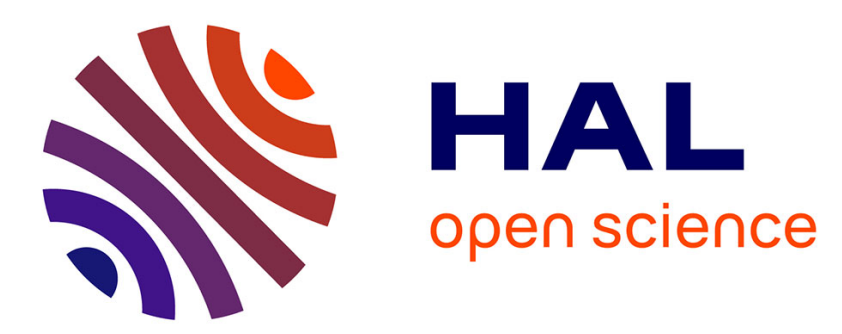

\title{
Comparison of Spatial Aloha and CSMA using Simple Stochastic Geometry Models for 1D and 2D Networks
}

Nadjib Achir, Younes Bouchaala, Paul Mühlethaler, Oyunchimeg Shagdar

\section{To cite this version:}

Nadjib Achir, Younes Bouchaala, Paul Mühlethaler, Oyunchimeg Shagdar. Comparison of Spatial Aloha and CSMA using Simple Stochastic Geometry Models for 1D and 2D Networks. ICT 2016 - 23rd International Conference on Telecommunications, 2016, May 2016, Thessalonique, Greece. 10.1109/ICT.2016.7500470 . hal-01368875

\section{HAL Id: hal-01368875 https://hal.inria.fr/hal-01368875}

Submitted on 20 Sep 2016

HAL is a multi-disciplinary open access archive for the deposit and dissemination of scientific research documents, whether they are published or not. The documents may come from teaching and research institutions in France or abroad, or from public or private research centers.
L'archive ouverte pluridisciplinaire HAL, est destinée au dépôt et à la diffusion de documents scientifiques de niveau recherche, publiés ou non, émanant des établissements d'enseignement et de recherche français ou étrangers, des laboratoires publics ou privés. 


\title{
Comparison of Spatial Aloha and CSMA using Simple Stochastic Geometry Models for 1D and 2D Networks
}

\author{
Nadjib Achir ${ }^{\dagger \S}$, Younes Bouchaala ${ }^{\dagger *}$, Paul Muhlethaler ${ }^{\dagger}$, and Oyunchimeg Shagdar ${ }^{\ddagger *}$ \\ $\dagger$ EVA Team, ${ }^{\ddagger}$ RITS Team, - INRIA Paris Rocquencourt B.P. 105, 78153 Le Chesnay, France \\ Emails: \{nadjib.achir, younes.bouchaala, paul.muhlethaler, oyunchimeg.shagdar\}@inria.fr \\ *Institut VEDECOM, 77, rue des Chantiers, 78000 Versailles, France \\ Emails: \{younes.bouchaala, oyunchimeg.shagdar\}@vedecom.fr \\ $\ddagger$ Universite Paris 13, Sorbonne Paris Cite - L2TI, 99 Avenue J-B Clément, 93430 Villetaneuse, France \\ Email: nadjib.achir@univ-paris13.fr
}

\begin{abstract}
Spatial throughput (i.e. throughput with spatial reuse) is important with new types of networks such as vehicular, sensor and military networks. The aim of this study is to compute the spatial throughput of Aloha and CSMA using tools for stochastic geometry. Our network nodes will be modeled as elements of a Poisson Point Process (PPP) of a one- or twodimensional space. Spatial Aloha can be modeled easily, the transmitting nodes are just selected with a given transmission probability. In spatial CSMA the nodes with the smallest backoff counter in their neighborhood will be selected to transmit and thus we can use random marks to perform the selection. We use the two models we have built to compare the spatial density of successful transmissions of CSMA and Aloha. To carry out a fair comparison, we will optimize both schemes by adjusting their parameters. For spatial Aloha, we can adapt the transmission probability, whereas for spatial CSMA we have to find the suitable carrier sense threshold.

The results obtained show that CSMA, when optimized, outperforms Aloha for nearly all the parameters of the network model values and we evaluate the gain of CSMA over Aloha. We also find interesting results concerning the effect of the model parameters on the performance of both Aloha and CSMA. The closed formulas we have obtained provide immediate evaluation of performance, whereas simulations may take minutes to give their results. Even if Aloha and CSMA are old protocols, this comparison of spatial performance is new and provides interesting and useful results.
\end{abstract}

Keywords-CSMA, slotted and non-slotted Aloha, spatial performance, stochastic geometry

\section{INTRODUCTION}

From the $60 \mathrm{~s}$ to the $80 \mathrm{~s}$, most performance evaluations were performed on wired networks. At the end the twentieth century came the revolution of wireless networks and, in the domain of local area networks, the IEEE 802.11 standard also called WiFi. The dominant architecture of WiFi networks involves an access point. This node, which is generally connected to the Internet, exchanges packets with surrounding nodes which are linked to the access point. In each cell around an access point there is only one packet sent at each instant since all the nodes are within carrier sense range of the each other. More recently, great progress in wireless transmission technology has paved the way to much bigger networks with more massive transmission patterns. This phenomenon is known as spatial reuse. In VANETs, extending the networks along the roads leads to vast networks where spatial reuse must inevitably be present. Moreover, the density of the vehicles may vary greatly, which leads to further complications. The high density of communicating vehicles on a road using the IEEE 802.11p - a CSMA-based protocol - justifies the optimization of CSMA in networks with spatial reuse.

However, the access schemes used in these recent wireless networks also use the well-known Carrier Sense Multiple Access techniques (CSMA) as did the first Wireless LANs (WLANs) such as the IEEE 802.11 standard $^{1}$. Therefore, a deeper understanding of CSMA with spatial reuse is needed. Aloha [1] was introduced in the early 70s. This is the simplest medium access control scheme since each node in the network simply transmits its packet at random and re-transmits it if necessary. Aloha can also, like CSMA, be used in these more recent networks which use the spatial reuse capability of the radio medium.

The remainder of this paper is organized as follows. Section II briefly describes related work. Section III describes the model proposed to study spatial Aloha and CSMA and develops the corresponding analytical model. The results of the model evaluating the influence of the parameters are reported in Section IV. Finally Section V concludes the paper.

\section{RELATED WORK}

The first studies on Aloha and CSMA were carried out in the 1970s resulting in several seminal studies such as the papers by Abramson [1] and Kleinrock [2].

These two papers, and a great number of others using the same analytical model framework, assume that the shared radio medium can carry only one packet at a given time. This is a fundamental characteristic of these analyses. We will see below that a period of ten yearrs was necessary before

\footnotetext{
${ }^{1}$ CSMA is also used in IEEE 802.15.4, a widely used protocol for sensor networks.
} 
papers investigating models with concurrent transmission on the medium appeared.

The study by Kleinrock [2] was precise in its analysis of the carrier-sense effect but, the model of the back-off technique remained at a global level. It was only in 2000 that the paper by Bianchi [3] which proposed a Markov model for the transition between the back-off states of nodes in IEEE 802.11 networks made a significant step forward in the modeling of the CSMA back-off technique. However [3] still did not introduce concurrent transmissions.

The study of Aloha has also received interesting contributions such as the analysis of slotted Aloha and the proposal of a scheme to stabilize Aloha [4]. However these improvements still do not consider concurrent transmissions.

Initial studies dealing with spatial reuse in spatial networks appeared in articles devoted to Aloha such as [5] and [6] and a model for slotted Aloha [5] was introduced by Ghez, Verdu and Schwartz in 1988. Their model included multipacket reception capability and was, to our best knowledge, the first quantitative model of wireless networks with spatial reuse. In [6] Baccelli et al. revisited the model and demonstrated that the probability of successful transmission in an Aloha network with spatial reuse could be accurately computed if the distance between the transmitter and the receiver was known. If this distance is known, the density of successful transmissions can also be computed.

In Aloha networks the randomization of the transmitters is stateless and the pattern of the simultaneously transmitting nodes is simple to evaluate. This explains the relative simplicity of the evaluation of Aloha. Describing the set of simultaneous transmission is a far more complex task for CSMA than for Aloha. Although in [7] the mean number of transmissions with CSMA is computed in a random linear network of vehicles, the authors only take into account the nearest interferer. In contrast, the present study takes the entire interference into account. The pattern of simultaneous transmissions in CSMA was evaluated in [8] using the Matern selection process [9]. The authors in [10] employ a similar process to evaluate interference but fail to compute the network throughput which is the corner stone of this paper. The model which was presented in [8], and improved in [11], forms the basis of the model that we use and extend in this paper. To the authors' best knowledge, there is no recent work pursuing these studies.

Concerning the comparison of spatial CSMA and spatial Aloha, this paper is apparently among the very few papers to tackle this subject. [12] uses extensive simulations to evaluate performance whereas [13] focuses on the outage probability and does not compute the spatial throughput. [14] focuses on a novel power control protocol to improve the aggregate throughput of the network. In addition to using of stochastic geometry models, the present paper has the originality of focusing its evaluation on transmission over a distance which is the mean distance between a node and its closest neighbor.

\section{SYSTEM MODEL}

In our model, the network nodes are randomly distributed according to a Poisson Point Process $\Phi$. We denote by $\lambda$ the intensity of the process. In our study we consider a 2D infinite plane, $\mathcal{S}=\mathbb{R}^{2}$ or a $1 \mathrm{D}$ infinite line, $\mathcal{S}=\mathbb{R}$. The $2 \mathrm{D}$ model will be suitable for Mobile Ad-hoc NETworks (MANETs) and Wireless Sensor Networks (WSNs). The 1D model is more relevant for vehicular Ad-hoc NETworks (VANETs). We assume that the signal received in a transmission is the result of the combination of a random fading $F$ and a power-law in the distance decay $1 / r^{\beta}$ where $\beta$ is the decay factor and is generally between 2 and 6 . In this paper the fading will be Rayleigh i.e exponentially distributed with parameter $\mu$. Thus the signal received when the transmitter and the receiver are at distance $r$ from each-other is $F / l(r)^{2}$ with $l(r)=r^{\beta}$.

We use the well-accepted SINR (Signal over Interference and Noise Ratio) with a capture threshold T. In the following, we assume that there is no thermal noise (i.e. $W \equiv 0$ ). Dealing with thermal noise would be easy but is not done in this paper due to limitations of space. An even more realistic model than the SINR based on a graded SINR model using Shannon's law is possible in our framework though with an increased computational cost. This is left for further work because we consider that the SINR model is already a meaningful model.

This study assumes that each node in $\Phi$ has a receiver at distance $r$ from the transmitter. This distance will a typical distance of the PPP computed as the average distance to the closest neighbor ${ }^{3}$. We recall that this distance is $r=1 / \lambda$ in 1D network and $r=1 / \sqrt{\lambda}$ in $2 \mathrm{D}$ networks, see Figure 1 .

In this paper we adopt a model where the network nodes are saturated. This means that at each instant each node always has a pending packet.

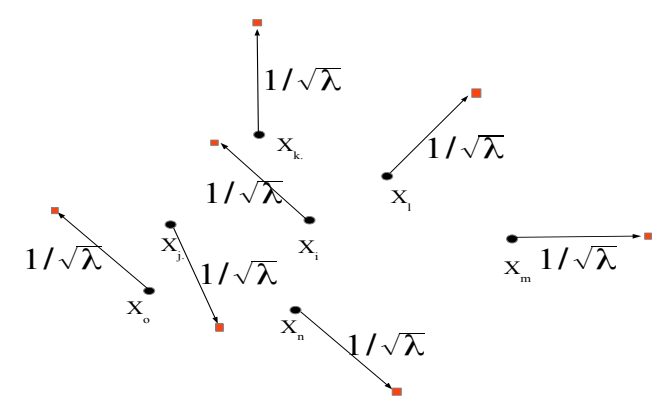

Fig. 1. Transmission to a typical node in a $2 \mathrm{D}$ network over a distance which is the average distance to the closest neighbor. .

\section{A. Analysis of spatial Aloha}

The analysis of spatial Aloha (slotted and non-slotted) comes respectively from [6] and [15]. In addition to the general network model described above, we need to add the nodes' transmission probability $p$. In slotted Aloha, $p$ denotes the node's transmission probability at each slot. In non-slotted Aloha and in a given node, the duration between the end of a packet transmission and the beginning of the next transmission is exponentially distributed with rate $\epsilon$. We can unify the notation with $p$ of the slotted model if we set $p=1 /\left(B+\frac{1}{\epsilon}\right)$

\footnotetext{
${ }^{2}$ The power received $P=\frac{P_{0} F}{l(r)}$ and we set $P_{0}=1$

${ }^{3}$ Dealing with a distance following the distribution of the n-th nearest neighbor [generalized gamma distribution] is possible in our frame but will incur more computation
} 
where $B$ denotes the duration of a packet ${ }^{4}$, see [15]. For nonslotted Aloha we use the rain model introduced in [15]. This model is shown to provide a very good estimation even when the nodes are fixed, as in the present model. For a complete presentation of the model for non-slotted Aloha the reader can refer to [15].

With Rayleigh fading, the capture probability is directly linked to the Laplace of the interference and of the noise, we have $p_{c}(r, \lambda)=\mathcal{L}_{I}(\mu T l(r)) \mathcal{L}_{W}(\mu T l(r))=$ $\mathcal{L}_{I}(\mu T l(r))(\operatorname{see}[6])$, with

$$
\mathcal{L}_{I}(s)=\exp \left(-\lambda p \int_{\mathcal{S}}\left(1-\mathcal{L}_{F}(s / l(|x|))\right) d x\right)
$$

and $\mathcal{L}_{F}($.$) is the Laplace transform of the fading.$

For the $2 \mathrm{D}$ and slotted Aloha case we have:

$$
p_{c}(r, \lambda)=\exp \left(\frac{-2 \pi^{2} \lambda p r^{2} T^{2 / \beta}}{\beta \sin (2 \pi / \beta)}\right),
$$

and for non-slotted Aloha:

$$
p_{c}(r, \lambda)=\exp \left(\frac{-4 \pi^{2} \lambda p r^{2} T^{2 / \beta}}{(\beta+2) \sin (2 \pi / \beta)}\right) .
$$

For the 1D and slotted Aloha case we have:

$$
p_{c}(r, \lambda)=\exp \left(\frac{-2 \pi \lambda p r T^{1 / \beta}}{\beta \sin (\pi / \beta)}\right),
$$

and for non-slotted Aloha:

$$
p_{c}(r, \lambda)=\exp \left(\frac{-4 \pi \lambda p r T^{1 / \beta}}{(\beta+2) \sin (\pi / \beta)}\right) .
$$

The density of successful transmissions is:

$$
D=\lambda p p_{c}(r, \lambda)
$$

The maximization of $D$ with respect to $p$ is straightforward. The table below provides the maximization (in $p$ ) of $D$ for slotted Aloha and non-slotted Aloha for 2D networks.

\begin{tabular}{|l|c|c|}
\hline 2D & $p_{o p t}$ & $D_{o p t}$ \\
\hline slotted Aloha & $\frac{\beta \sin (2 \pi / \beta)}{2 \pi^{2} \lambda r^{2} T^{2 / \beta}}$ & $\frac{\beta \sin (2 \pi / \beta) e^{-1}}{2 \pi^{2} r^{2} T^{2 / \beta}}$ \\
\hline non-slotted Aloha & $\frac{\sin (2 \pi / \beta)(\beta+2)}{4 \pi^{2} \lambda r^{2} T^{2 / \beta}}$ & $\frac{\sin (2 \pi / \beta)(\beta+2) e^{-1}}{4 \pi^{2} r^{2} T^{2 / \beta}}$ \\
\hline
\end{tabular}

The case of $1 \mathrm{D}$ networks is given in the following table :

\begin{tabular}{|l|c|c|}
\hline 1D & $p_{o p t}$ & $D_{o p t}$ \\
\hline slotted Aloha & $\frac{\beta \sin (\pi / \beta)}{2 \pi \lambda r T^{1 / \beta}}$ & $\frac{\beta \sin (\pi / \beta) e^{-1}}{2 \pi r T^{1 / \beta}}$ \\
\hline non-slotted Aloha & $\frac{\sin (\pi / \beta)(\beta+2)}{4 \pi \lambda r T^{1 / \beta}}$ & $\frac{\sin (\pi / \beta)(\beta+2) e^{-1}}{4 \pi r T^{1 / \beta}}$ \\
\hline
\end{tabular}

The computations in the above tables assume that values of $p_{\text {opt }}$ are smaller than or equal to 1 . This will be the case for the values of the system used in Section IV. If it is not the case, then $p_{o p t}=1$ and $D_{o p t}$ is obtained using the first four formulas of the above section setting $p=1$ in $p_{c}(r, \lambda)$.

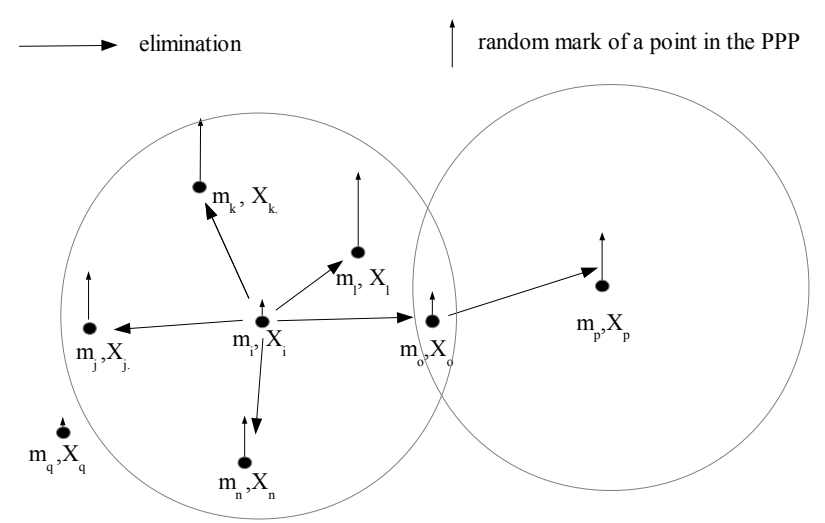

Fig. 2. Matern CSMA selection process and an example of over-elimination.

\section{B. Analysis of spatial CSMA}

To mimic the CSMA selection process, we use a Matern process in a marked PPP: $\left(X_{i} \in \Phi, m_{i}\right)$, as is done in [11]. The $m_{i}$ are thus random numbers in $[0,1]$. We denote by $F_{i, j}$ the fading for the transmission between $X_{i}$ and $X_{j}$ The Matern selection process elects the the points $X_{k}$ with the smallest random marks $m_{k}$ in their neighborhood. We need to introduce the carrier sense threshold $P_{c s}$ to precisely define the neighborhood of a point $X_{i}$. We define $\mathcal{V}\left(X_{i}\right)=\left\{X_{j} \in\right.$ $\left.X_{i} F_{i, j} / l\left(\left|X_{i}-X_{j}\right|\right)>P_{c s}\right\}$ the neighborhood of $X_{i}$. A node $X_{i}$ will be selected in the Matern selection process if and only if $\forall X_{j} \in \mathcal{V}\left(X_{i}\right) m_{i}<m_{j}$. As can seen in Figure 2, $X_{i}$ has the smallest mark $m_{i}$ in its neighborhood and thus it will be selected in the Matern selection process. We observe that $X_{q}$ does in fact have a smaller mark, but it is not within node $i$ 's neighborhood. For the sake of simplicity, we have not taken into account any Rayleigh fading $(F \equiv 1)$ in Figure 2 and thus the neighborhoods of the nodes are discs of the same radius. We can observe that the Matern selection process results in an over-elimination of nodes. When one node is eliminated by a node with a smaller mark, the node which has been eliminated should not continue to eliminate other nodes. But this overelimination can occur, as is shown in Figure 2 where node $O$ is eliminated by node $i$, but node $o$ goes on to eliminate node $p$ in the Matern selection process, whereas in a real CSMA system, node $o$ is correctly eliminated by node $i$, but, being eliminated, node $o$ can not eliminate another node. We do not take this case into account in our model.

We note the medium access indicator of node $X_{i} e_{i}=$ $\mathbb{I}\left(\forall X_{j} \in \mathcal{V}\left(X_{i}\right) m_{i}<m_{j}\right)$

Proposition 1. The mean number of neighbors of a node is:

$$
\mathcal{N}=\lambda \int_{\mathcal{S}} P\left\{F \geqslant P_{c s} l(|x|)\right\} d x .
$$

In a $2 D$ network we have :

$$
\mathcal{N}=\frac{2 \pi \lambda \Gamma(2 / \beta)}{\beta\left(P_{c s} \mu\right)^{2 / \beta}}
$$

\footnotetext{
$4 \frac{1}{\epsilon}$ is the mean duration between the end of a packet and the start time of the next packet.
} 
In a $1 D$ network we have :

$$
\mathcal{N}=\frac{\lambda \Gamma(1 / \beta)}{\beta\left(P_{c s} \mu\right)^{1 / \beta}} .
$$

This result is very simple. Let $F_{j}^{0}$ be the fading between the node at the origin $X_{i}$ and node $X_{j}$

This is just the application of Slivnyak's theorem and Campbell's formula, see [16], [11]

$$
\begin{aligned}
\mathcal{N} & = & E^{0}\left[\sum_{X_{j} \in \phi} \mathbb{I}\left(F_{j}^{0} l\left(\left|X_{j}-X_{i}\right|\right) \geqslant P_{c s}\right]\right. \\
& = & \lambda \int_{\mathcal{S}} P\left\{F \geqslant P_{c s} l(|x|)\right\} d x
\end{aligned}
$$

A straightforward computation provides the explicit value of $\mathcal{N}$ in $1 \mathrm{D}$ and $2 \mathrm{D}$ cases.

Proposition 2. The probability ${ }^{5} p$ that a given node $X_{0}$ transmits i.e. $e_{0}=1$ is:

$$
p=\mathbf{E}^{0}\left[e_{0}\right]=\frac{1-e^{-\mathcal{N}}}{\mathcal{N}} .
$$

Proof: The proof is obtained by computing the probability that a given node at the origin with a mark $m=t$ is allowed to transmit. The result is then obtained by deconditioning on $t$. The details of the proof can be found in [11].

Proposition 3. The probability that $X_{0}$ transmits given that there is another node $X_{j} \in \Phi$ at distance $r$ is $p_{r}$ with

$$
p_{r}=p-e^{-P_{c s} \mu l(r)}\left(\frac{1-e^{-\mathcal{N}}}{\mathcal{N}^{2}}-\frac{e^{-\mathcal{N}}}{\mathcal{N}}\right) .
$$

Proof: The proof is the same as that of Proposition 2.

Proposition 4. Let us suppose that $X_{1}$ and $X_{2}$ are two points in $\Phi$ such that $\left|X_{1}-X_{2}\right|=r$. We suppose that node $X_{2}$ is retained by the selection process. The probability that $X_{1}$ is also retained is:

$$
h(r)=\frac{\frac{2}{b(r)-\mathcal{N}}\left(\frac{1-e^{-\mathcal{N}}}{\mathcal{N}}-\frac{1-e^{-b(r)}}{b(r)}\right)\left(1-e^{-P_{c s} \mu l(r)}\right)}{\frac{1-e^{-\mathcal{N}}}{\mathcal{N}}-e^{-P_{c s} \mu l(r)}\left(\frac{1-e^{-\mathcal{N}}}{\mathcal{N}^{2}}-\frac{e^{-\mathcal{N}}}{\mathcal{N}}\right)}
$$

with

$$
b(r)=2 \mathcal{N}-\lambda \int_{\mathcal{S}} e^{-P_{c s} \mu(l(|x|)+l(|r-x|)} d x .
$$

In a $2 D$ network, we have:

$$
b(r)=2 \mathcal{N}-\lambda \int_{0}^{\infty} \int_{0}^{2 \pi} e^{-P_{c s} \mu\left(l(\tau)+l\left(\sqrt{\tau^{2}+r^{2}-2 r \tau \cos (\theta)}\right)\right)} d \tau d \theta .
$$

In a $1 D$ network, we have:

$$
b(r)=2 \mathcal{N}-\lambda \int_{-\infty}^{\infty} e^{-P_{c s} \mu(l(\tau)+l(|r-\tau|))} d \tau .
$$

\footnotetext{
${ }^{5}$ We use $p$ here, the same notation as in Aloha since the two parameters have the same meaning. In Aloha $p$ is chosen by the user and is the transmission probability. In CSMA $p$ is the probability that a node transmits, which is governed by the carrier sense mechanism and thus $p$ is a function of $P_{c s}$.
}

Proof: The proof can be found in [11].

Proposition 5. The probability of successfully receiving a packet at distance $r$ in a CSMA system (carrier sense threshold $P_{c s}$ modeled by a Matern selection process) and with a capture threshold $T$ is:

$$
p_{c}\left(r, P_{c s}\right) \simeq \exp \left(-\lambda \int_{\mathcal{S}} \frac{h(|x|)}{1+\frac{l(|x-r|)}{T l(r)}} d x\right) .
$$

In a $2 D$ network, we have:

$$
\begin{aligned}
& \text { In a } 2 D \text { network, we have: } \\
& p_{c}\left(r, P_{c s}\right) \simeq \exp \left(-\lambda \int_{0}^{\infty} \int_{0}^{2 \pi} \frac{\tau h(\tau)}{1+\frac{l\left(\sqrt{\tau^{2}+r^{2}-2 r \tau \cos (\theta)}\right)}{T l(r)}} d \tau d \theta\right)
\end{aligned}
$$

In a $1 D$ network, we have;

$$
\begin{aligned}
& \text { network, we have; } \\
& p_{c}\left(r, P_{c s}\right) \simeq \exp \left(-\lambda \int_{-\infty}^{\infty} \frac{h(\tau)}{1+\frac{l(|r-\tau|)}{T l(r)}} d \tau\right)
\end{aligned}
$$

Proof: The idea of the proof is to consider a transmitter at the origin and to compute the probability of successful reception by a receiver at distance $r$. We assume the presence of another transmitting node at distance $\tau$. According to proposition 4 , the density of such nodes is $\lambda h(\tau)$. We approximate the interference by the interference of a Poisson Process of density $\lambda h(\tau)$. The result is obtained by integrating on $\tau$. The details of the proof can be found in [11].

Proposition 6. The spatial density of successful transmissions is thus:

$$
D=\lambda p p_{c}\left(r, P_{c s}\right) \text {. }
$$

This spatial density has a $1 D$ and a $2 D$ version and the values of $p$ and $p_{c}\left(r, P_{c s}\right)$ are chosen accordingly.

Proof: Proposition 6 is just the exploitation of propositions 2 and 5.

\section{RESUlts OF THE MODEL}

In this section, we use the model to analyze the network performance and the influence of the model's parameters. We study the transmissions for pairs of source-destination nodes at distance $r . r$ is set at $1 / \sqrt{\bar{\lambda}}$ or $1 / \lambda$ for 2D and 1D networks respectively. $r$ can be seen as a typical distance in these networks since it is the average distance between a node and its closest neighbor. Thus the transmitters are in the Poisson Point Process and for each transmitter, we create a random receiver at distance $r$.

A. Optimizing the density of successful transmissions with $p$ for Aloha and the carrier sense threshold $P_{c s}$ for CSMA

In this section we fix the parameters of the model $\lambda, T$ and $\mu$.

Figure 3 illustrates the density of successful transmissions for both slotted and non-slotted Aloha. This density starts to increase, reaches a maximum for a value of the transmission probability $p_{\text {opt }}$ computed in Section III-A, and then decreases.

For CSMA we vary $P_{c S}$ to maximize the density of successful transmissions. The probability of transmission $p$ in CSMA increases when $P_{c s}$ increases, which is because with larger values of $P_{c s}$ transmission is easier. But, in contrast, when $P_{c s}$ increases, $p_{c}\left(r, P_{c s}\right)$ decreases, as can be shown using the equation of proposition 4 . When $P_{c s}$ increases, $h(\tau)$ increases and thus $p_{c}\left(r, P_{c s}\right)$ decreases. Thus there is an optimal value of $P_{c s}$ which optimizes the density of successful transmissions. Studying some significant examples, we have 
seen that the density of successful transmissions always has the same behavior, as shown in Figure 3. For small values of $P_{c s}$ and when we increase $P_{c s}, p$ increases faster than $p_{c}\left(r, P_{c s}\right)$ decreases, and thus the density of successful transmissions is an increasing function of $P_{c s}$. This density reaches a maximum for a given value of $P_{c s}$ and then becomes a decreasing function of $P_{c s}$. We assume that this is always the case although it seems difficult to prove it in the equations. We use Maple to numerically compute this optimum of the density of successful transmissions.

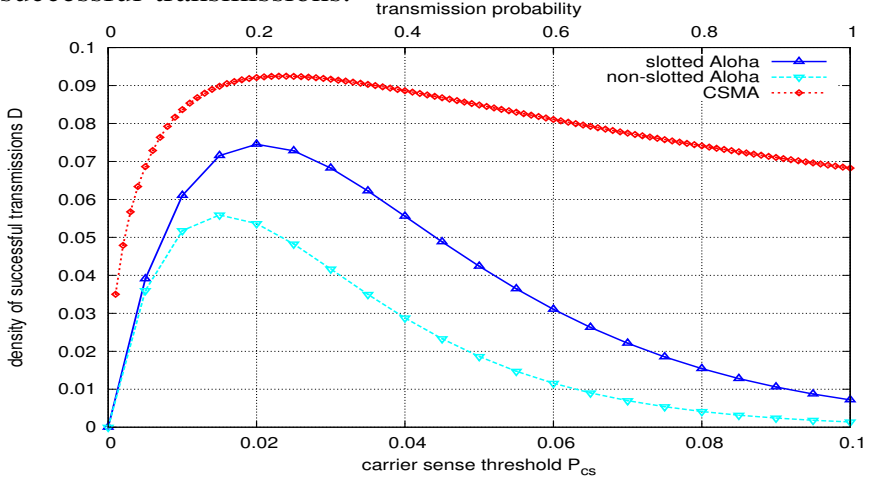

Fig. 3. Density of successful transmissions versus carrier threshold ( $T=1$, $\mu=10, \beta=4)$.

\section{B. Effect of the fading rate $\mu$}

For Aloha, computing the probability of successful transmissions and the density of successful transmissions shows that the fading rate $\mu$ disappears in the equations ${ }^{6}$. Thus this parameter has no effect on the performance of Aloha (slotted and non-slotted). For CSMA we observe that in the probability of transmission $p$ found in Proposition 2, we can isolate $\mu P_{c s}$. It is the same for $p_{c}\left(r, P_{c s}\right)$. Thus if we multiply $\mu$ by $x$, we can obtain the same performance by dividing $P_{c s}$ by $x$. Thus $\mu$ does not influence the global performance of the system; the optimum density of successful transmissions, the probability of capture $p_{c}\left(r, P_{c s}\right)$ and the probability of transmission $p$ at the optimum value of $P_{c s}$.

\section{Effect of the density of nodes $\lambda$}

For both slotted and non-slotted Aloha, the maximum density of successful transmissions is obtained by we exploiting formulas of Section III-A.

We study transmissions over the average distance to the closest neighbor, thus $\lambda r=1$ for $1 \mathrm{D}$ networks and $\lambda r^{2}=1$ for $2 \mathrm{D}$ networks. We use the following parameters $T=1$, $\mu=10$ and $\beta=4$. In the above formula, we observe that the maximum density of successful transmissions is linear in $\lambda$ for 1D and 2D networks, as is also shown in Figures 4 and 5.

For CSMA, we compute the optimum density of successful transmissions when $P_{c s}$ is optimized versus $\lambda$ the density of nodes in the network. The results of these computations are shown in Figure 4 for 2D networks and in Figure 5 for 1D networks. Our numerical study shows that the density of successful transmissions is linear in $\lambda$. This means that the maximum of the product of $p p_{c}\left(r, P_{c s}\right)$ does not depend on

\footnotetext{
${ }^{6}$ This because the fading affects the transmission and the interference equally.
}

$\lambda$. This is an interesting result and one which is not easily apparent in the analytical formulas of $p p_{c}\left(r, P_{c s}\right)$.

Figures 4 and 5 also show the density of successful transmissions for CSMA when the carrier sense threshold is constant and taken as the optimal value for $\lambda=1$. The loss is already significant for small values of $\lambda: 26 \%$ for $\lambda=0.1$ and is even more significant for large values of $\lambda: 80 \%$ for $\lambda=10$ in $2 \mathrm{D}$ networks and $85 \%$ for $\lambda=10$ in $1 \mathrm{D}$ networks. For instance, this means that, in a VANET, the channel cannot be used efficiently if the carrier sense threshold is not properly optimized according to the density of vehicles. When $\lambda=0.1$ and if we use the optimization for $\lambda=1$ we do not have any restriction on the transmission rights but rather an excess of transmission rights. The problem arises from the probability of success for a given transmission. When $\lambda=10$ and if we use the optimization for $\lambda=1$ we have a tight restriction on the transmission rights, whereas a given transmission is very well protected by the CSMA scheme and thus every transmission is nearly always successful. The model shows that the problem concerning the access right is much more detrimental to the global throughput than collisions would have been if the density of nodes had been overestimated.

We have studied the probability of capture when the throughput is optimized. We observed that, for CSMA, the optimum throughput is not obtained when most of the transmissions are successful but rather when the success rate is around $55 \%$ in 2D networks and around $70 \%$ in 1D networks. The numerical results we obtained show that, at the optimum, $p_{c}\left(r, P_{c s}\right)$ does not depend on $\lambda$ and we also deduce that $p$ does not depend on $\lambda$. This is an interesting result which is not brought to light using the analytical formulas. In Aloha the probability of capture when the throughput is optimized is $1 / e$ as shown by the computations in Section III-A.

When we compare the density of successful transmissions for Aloha with the density of successful transmissions for CSMA, we find that the gain of CSMA is 57\% and 78\% with non-slotted and $18 \%$ and $33 \%$ with slotted Aloha for 2D and 1D networks respectively, see Figures 4 and 5. We observe that this gain is particularly significant in 1D networks. However, when the carrier sense threshold is not optimized, Aloha can offer a larger density of successful transmissions, as shown in Figures 4 and 5. A very useful property of Aloha is that when we consider transmissions over the average distance to the closest neighbor, the probability which optimizes the density of successful transmissions does not depend on $\lambda$ (see the formulas for $p_{\text {opt }}$ in Section III- $\mathrm{A}^{7}$ ). Thus in contrast to CSMA, there is no risk of an unsuitable tuning for Aloha. This represents a huge advantage of Aloha. In a VANET, where the density of vehicles may greatly vary, we may wonder whether Aloha would not be a better option than CSMA if we can not dynamically adjust the carrier threshold.

\section{Effect of the capture threshold $T$}

We study the effect of the capture threshold on the maximum density of successful transmissions. In Figure 6 and Figure 7 we plot the maximum density of successful transmissions for $T$ varying from 0.01 to 10 respectively for

\footnotetext{
${ }^{7}$ this is because in $1 \mathrm{D}$ networks $\lambda r=1$ and in $2 \mathrm{D}$ networks $\lambda r^{2}=1$
} 


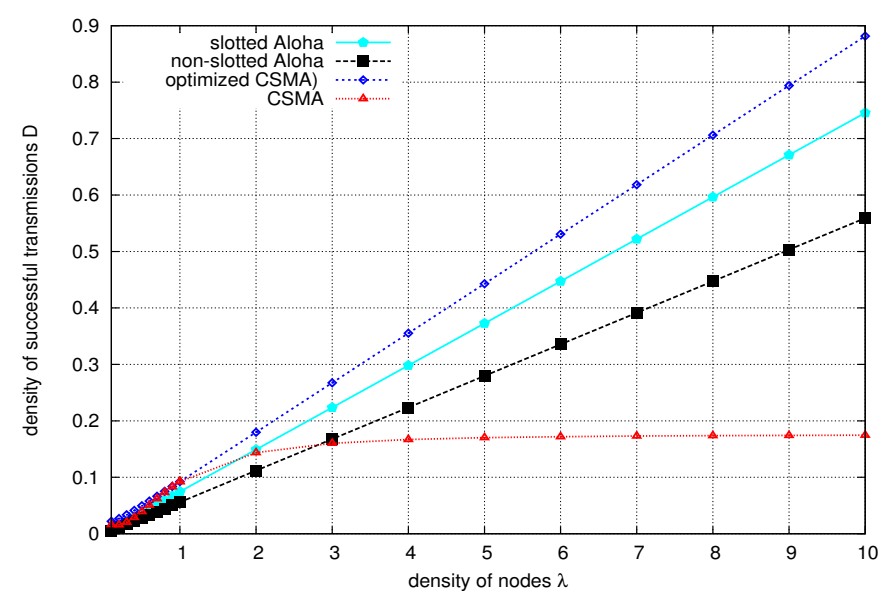

Fig. 4. Density of successful transmissions versus density of nodes $(T=1$, $\mu=10, \beta=4)$. Spatial network (2 D)

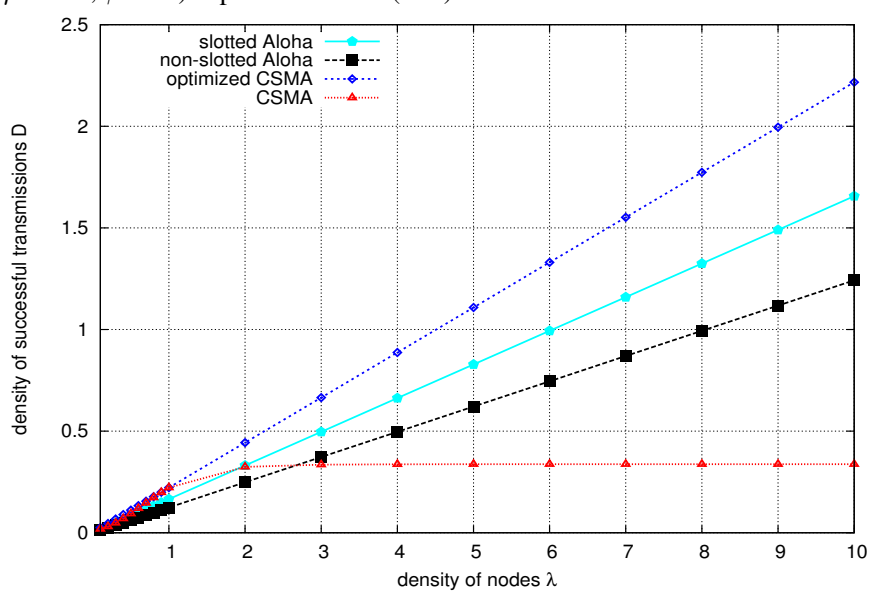

Fig. 5. Density of successful transmissions versus density of nodes ( $T=1$, $\mu=10, \beta=4)$. Linear network (1 D)

2D and 1D networks. We observe that dividing the capture threshold by 100 leads to multiplying the density of successful transmissions by 5.6 and 1.9 for 2D and 1D CSMA networks. For slotted and non-slotted Aloha, the density of successful transmissions is multiplied by 10 and 3.16 for 2D and 1D networks respectively. This means that a small capture threshold is much more beneficial in 2D networks. Our study of the analytical model does not show any obvious scaling of the density of successful transmissions with the capture threshold $T$ in CSMA networks. For Aloha (slotted and non-slotted) the scaling is respectively $1 / T^{2 / \beta}$ and $1 / T^{1 / \beta}$. For $T=10$, CSMA outperforms slotted and non-slotted Aloha by $68 \%$ and $124 \%$ in $2 \mathrm{D}$ networks. For $T=10$, CSMA outperforms slotted and non-slotted Aloha by $65 \%$ and $119 \%$ in 1D networks

\section{E. Effect of the transmission decay $\beta$}

In Figures 8 and 9, we plot the maximum density of successful transmissions for $\beta$ varying from 2 to 6 respectively for $2 \mathrm{D}$ and $1 \mathrm{D}$ networks.

In 2D CSMA networks, we observe that the maximum density of successful transmissions is multiplied by 1.91 when $\beta$ varies from 2.5 to 6 . For linear (1D) CSMA networks the maximum density of successful transmissions is multiplied by 1.32 when $\beta$ varies from 2 to 6 . As for the capture threshold, the effect of a large transmission decay is less beneficial for

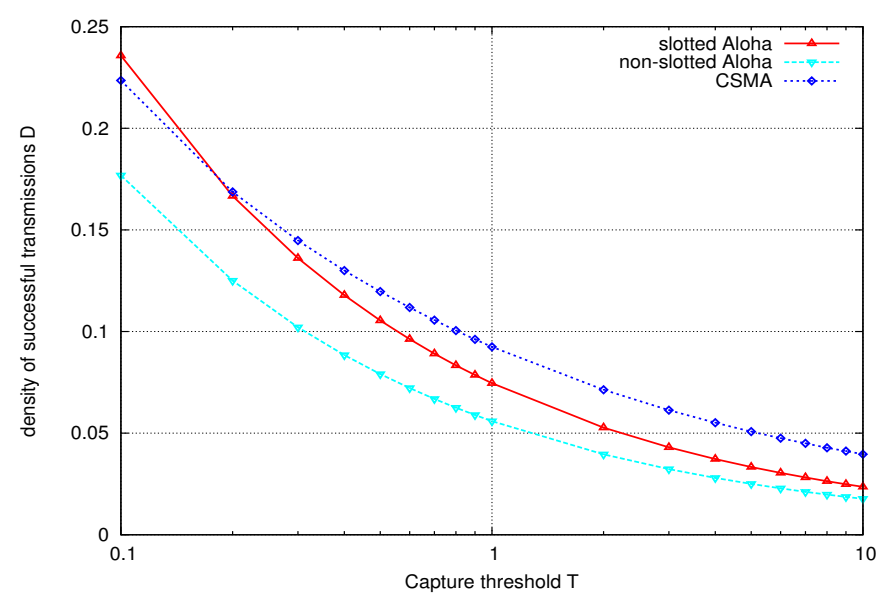

Fig. 6. Density of successful transmissions versus capture threshold $T$ for $2 \mathrm{D}$ networks $(\lambda=1, \mu=10, \beta=4)$.

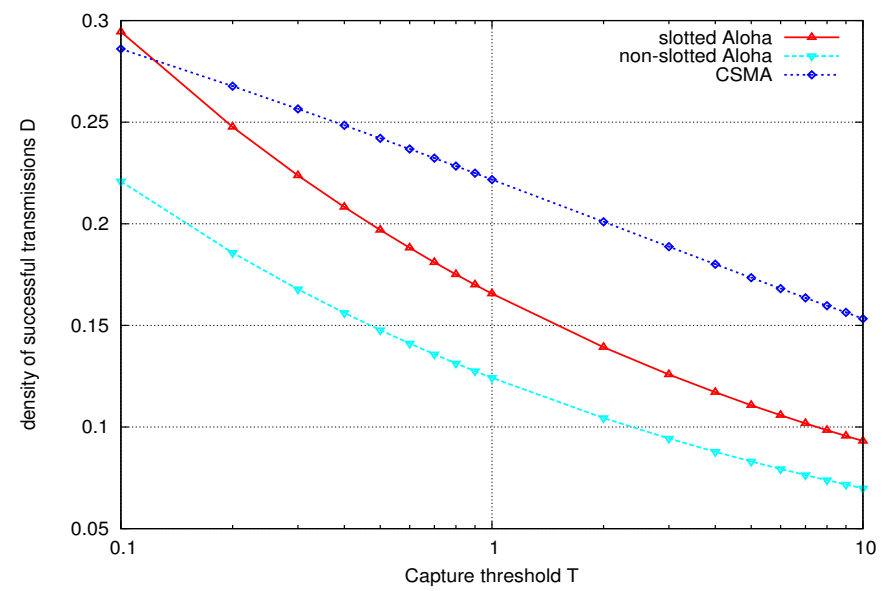

Fig. 7. Density of successful transmission versus the capture threshold $T$ $(\lambda=1, \mu=10, T=1)$. Linear network (1 D)

1D networks than for 2D networks. Our study does not show any apparent scaling of the density of successful transmissions with the capture threshold $\beta$.

In 2D Aloha networks, we observe that the maximum density of successful transmissions is multiplied by 3.54 and 2.62 for slotted and non-slotted Aloha when $\beta$ varies from 2.5 to 6 . In $1 \mathrm{D}$ Aloha networks, the maximum density of successful transmissions is multiplied by 1.5 and 1.0 for slotted and non-slotted Aloha when $\beta$ varies from 2 to 6 . The scaling of Aloha's performance with $\beta$ is given by the formulas in Section IN-A.CONCLUSION

In this paper, we present spatial models for Aloha and CSMA networks. These models providing closed formulas result in instantaneous performance evaluation of the two schemes. We show the importance of optimizing tuning parameters according to the network parameters. We have shown that the optimized density of successful transmissions (when transmissions over the average distance to the closest neighbor are considered) scales linearly with the density of the nodes, both for Aloha and CSMA networks. However, in CSMA the carrier sense threshold must be adapted to the density of the nodes, whereas in Aloha a constant transmission probability can be used. In CSMA, using a constant carrier threshold leads 


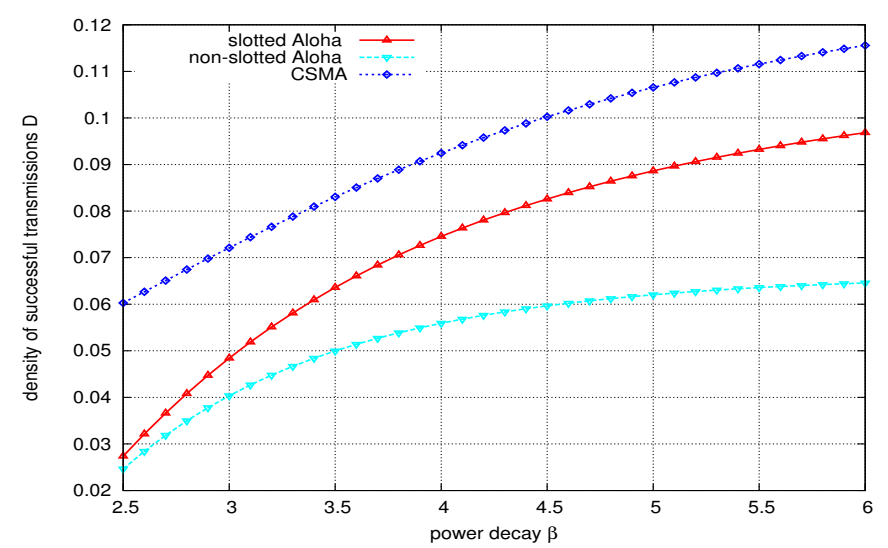

Fig. 8. Density of successful transmissions versus the decay factor $\beta$ for $2 \mathrm{D}$ networks $(\lambda=1, \mu=10, T=1)$.

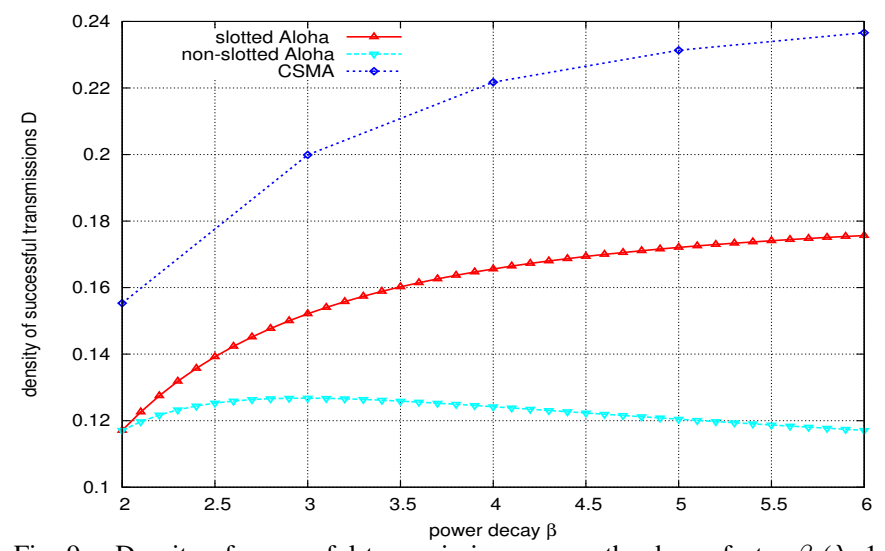

Fig. 9. Density of successful transmissions versus the decay factor $\beta$ ( $\lambda=1$, $\mu=10, T=1$ ). Linear network (1 D)

to a very significant loss in the network's global throughput. This effect is much more detrimental when the density of the nodes in the network is underestimated than when it is overestimated.

For Aloha networks, when we study transmissions over the average distance to the closest neighbor, the optimization does not depend on the density of nodes, which is a very interesting property. Thus in Aloha networks, the density of successful transmissions easily scales linearly in $\lambda$ when we vary $\lambda$ whereas in CSMA networks the protocol must be carefully tuned to obtain this scaling.

However, CSMA (when optimized) outperforms Aloha for nearly all values of the model's parameters. For instance, for $\lambda=1$ and $T=1$ the improvement brought about by CSMA compared to Aloha is notable. This improvement is very significant for $\lambda=1$ and $T=10$.

Our models allow the influence of the network model's parameters to be studied qualitatively. For instance, the improvement in the performance can be accurately computed when $T$ is decreased or when $\beta$ is increased. The models show that the influence of $T$ and $\beta$ on the performance is more pronounced for 2D than for 1D networks.

The models for Aloha have already been compared with simulation results. This comparison shows a very good match- ing between the model and the simulation results. This is because there is no approximation made in the Aloha model. In contrast, there are a number of approximations made in the CSMA model such as the selection process and the back-off scheme. However, initial simulations suggest that the CSMA model captures the essential behavior of the carrier sense technique and provides reasonably accurate results.

\section{REFERENCES}

[1] N. Abramson, "The aloha system: Another alternative for computer communications," in Proceedings of the November 17-19, 1970, Fall Joint Computer Conference, ser. AFIPS '70 (Fall). New York, NY, USA: ACM, 1970, pp. 281-285. [Online]. Available: http://doi.acm.org/10.1145/1478462.1478502

[2] L. Kleinrock and F. Tobagi, "Packet switching in radio channels: Part I-carrier sense multiple-access modes and their throughput-delay characteristics," IEEE Transactions on Communications, vol. COM23, no. 12, pp. 1400-1416, December 1975, (Also, "Multiple Access Communications, Foundations for Emerging Technologies", Norman Abramson (Ed), IEEE Press, 1992, pp. 272-288.).

[3] G. Bianchi, "Performance Analysis of the IEEE 802.11 Distributed Coordination Function," IEEE Journal of Selected Areas in Communications., vol. 18, no. 3, pp. 535-547, March 2000. [Online]. Available: http://dx.doi.org/10.1109/49.840210

[4] R. Rivest, "Network control by bayesian brodcast," in Report MIT/LCS/TM-285. Cambridge, MA: MIT, Laboratory for Computer Science, 1985.

[5] S. Ghez, S. Verdu, and S. Schartz, "Stability properties of slotted Aloha with multipacket reception capability," IEEE Trans. Automat. Contr., vol 7, pp. 640-648, 1988.

[6] F. Baccelli, B. Blaszczyszyn, and P. Muhlethaler, "An aloha protocol for multihop mobile wireless networks," Information Theory, IEEE Transactions on, vol. 52, no. 2, pp. 421-436, Feb 2006.

[7] P. Jacquet and P. Muhlethaler, "Mean number of transmissions with csma in a linear network," in 2010 IEEE 72nd Vehicular Technology Conference: VTC2010-Fall, 69 September 2010, Ottawa, Canada 2010.

[8] P. Muhlethaler and A. Najid, "Throughput optimization in multihop csma mobile ad hoc networks," in EW 2004. The 5th European Wireless Conference, February 24 - 27. Barcelona 2004.

[9] D. Stoyan, W. S. Kendall, and J. Mecke, Stochastic geometry and its applications. 2nd edition. Wiley, 1995.

[10] A. Busson and G. Chelius, "Point processes for interference modeling in csma/ca ad-hoc networks," in Conference: Proceedings of the 6th ACM International Workshop on Performance Evaluation of Wireless Ad Hoc, Sensor, and Ubiquitous Networks, PE-WASUN 2009, October 28-29 2009, Tenerife, Canary Islands, Spain, 2009.

[11] F. Baccelli and B. Błaszczyszyn, Stochastic Geometry and Wireless Networks, Volume II - Applications, ser. Foundations and Trends in Networking. NoW Publishers, 2009, vol. 4, No 1-2.

[12] B. Blaszczyszyn, P. Muhlethaler, and S. Banaouas, "Comparison of the maximal spatial throughput of Aloha and CSMA in Wireless multihop Ad-Hoc Networks," in Wireless Ad-Hoc Networks, H. Zhou, Ed. InTech, Dec. 2012, pp. 3-22. [Online]. Available: https://hal.inria.fr/inria-00530093

[13] M. Kaynia and N. Jindal, "Performance of aloha and csma in spatially distributed wireless networks," in Proceedings of ICC 2008, 19-23 May 2008, Beijing, China 2008.

[14] R. Manikandan and K. Selvakumar, "Throughput performance of aloha and csma mac protocol in power for wireless ad hoc networks," International Journal of Computer and Information Technology (ISSN: 2279 0764) Volume 02 Issue 04, July 2013.

[15] B. Blaszczyszyn and P. Muhlethaler, "Interference and SINR coverage in spatial non-slotted aloha networks," annals of telecommunications, arXiv admin note: substantial text overlap with arXiv:1002.1629. [Online]. Available: https://hal.inria.fr/hal-01082772

[16] F. Baccelli and B. Błaszczyszyn, Stochastic Geometry and Wireless Networks, Volume I - Theory, ser. Foundations and Trends in Networking. NoW Publishers, 2009, vol. 3, No 3-4. 